\title{
Adequacy of Vancomycin/Gentamicin as Empirical Regimen in Treatment of Late Onset Sepsis: Retrospective Study in Neonatal Intensive Care Unit in UAE
}

\author{
Aisha Al Khaaldi ${ }^{1}$, Yasmin Karahamo ${ }^{2}$, Eman Qadoom ${ }^{3}$, Majid Al Teneiji ${ }^{4}$, Tahra AlAil ${ }^{2}$, Amna Al \\ Kuwaiti $^{5}$, Alzubair Almalik ${ }^{6}$, Tasnim Dawoud ${ }^{3}$ and Hossam Al-Tatari ${ }^{1 *}$ \\ ${ }^{1}$ Pediatric Infectious Disease Division, Tawam Hospital, United Arab Emirates \\ ${ }^{2}$ Pediatric Residency Program, Tawam Hospital, United Arab Emirates
}

${ }^{3}$ Clinical Pharmacy Department, Oasis Hospital, United Arab Emirates

${ }^{4}$ Pediatric Pulmonology Division, Tawam Hospital, United Arab Emirates

${ }^{5}$ Pediatric Allergy and Immunology, Tawam Hospital, Al Ain, United Arab Emirates

${ }^{6}$ Medical Intern, Tawam Hospital, Al Ain, United Arab Emirates

${ }^{*}$ Corresponding author: Hossam Al-Tatari, Division of Pediatric Infectious Diseases, Tawam Hospital, UAE, Tel: +971506203271; E-mail: htatari@seha.ae

Received date: March 13, 2017; Accepted date: April 19, 2017; Published date: April 24, 2017

Citation: Al Khaaldi A, Karahamo Y, Qadoom E, Al Teneiji M, AlAil T, et al. (2017) Adequacy of Vancomycin/Gentamicin as Empirical Regimen in Treatment of Late Onset Sepsis: Retrospective Study in Neonatal Intensive Care Unit in UAE. Pediatric Infect Dis 2: 41. doi: $10.21767 / 2573-0282.100041$

Copyright: (C) 2017 Al Khaaldi A, et al. This is an open-access article distributed under the terms of the Creative Commons Attribution License, which permits unrestricted use, distribution, and reproduction in any medium, provided the original author and source are credited.

\begin{abstract}
Despite the constant look out for microbial patterns and antibiotic sensitivities, neonatal sepsis still has high morbidity and mortality if not managed appropriately. The local biograms differ from region to region, and there has been a major shift of empirical antimicrobial treatment for late onset neonatal sepsis (LOS) worldwide. Our study was based on positive cultures obtained from neonates admitted to our Neonatal Intensive Care Unit (NICU) with the diagnosis of possible sepsis between $\geq 72 \mathrm{hrs}$ and 90 days of age. The most common causative organisms in our patients were Coagulase Negative Staphylococci (CONS), Staphylococcus aureus, Klebsiella pneumoniae, E.coli and Pseudomonas. Our current empirical regimen for LOS consists of Vancomycin and Gentamicin. Assessment of our regimen's adequacy revealed $100 \%$ sensitivity to Vancomycin among gram positive organisms and $95.5 \%$ sensitivity to Gentamicin among gram negative organisms. The local biograms must be revisited from time to time to optimize empirical antibiotic choices and ensure a better quality of care for our patients.
\end{abstract}

Keywords: Vancomycin; Gentamicin; Neonatal intensive care unit; Neonatal sepsis; Staphylococci; Infections

\section{Introduction}

Late onset neonatal sepsis (LOS) is a hospital acquired Infection with a substantial rate of morbidity and mortality. LOS is defined as signs consistent with sepsis that occur from 72 hours of life, with some references defining it as starting from 7 days of life until the end of the third month of life [1-3]. The estimated incidence is $25 \%$ among singleton births and $22.6 \%$ among multiple births of very low birth weight infants [4]. Peak Incidence reported between the $10^{\text {th }}$ and $22^{\text {nd }}$ day of life [4]. Organisms that have been implicated in causing LOS include Coagulase Negative Staphylococci (CONS), Staphylococcus aureus, enteric gram negative rods, Pseudomonas aeruginosa, Enterococcus species, Group B Streptococci and Candida species [5].

Signs of LOS are vague, therefore close monitoring and a high level of clinical suspicion in the NICU setting is paramount, this coupled with a coherent understanding of the epidemiology and the antimicrobial sensitivity pattern allows prompt management of such infections.

With those objectives in mind, we conducted this study to:

- Study the most common causative organisms of LOS in the NICU at Tawam Hospital, Al Ain, United Arab Emirates (UAE). The NICU at Tawam Hospital is a level III referral unit that serves the emirate of Abu Dhabi and parts of the northern region of the UAE.

- Look at the adequacy of our current empirical antibiotic regimen that consists of Vancomycin and Gentamicin in covering those organisms.

\section{Methods}

Data was collected in retrospective cohort fashion from medical charts of infants with proven LOS between January 2011 and June 2015. Our inclusion criteria consisted of patients who exhibited signs of sepsis between the age $\geq 72$ hours and 90 days; and had positive cultures obtained either from blood, 
urine, cerebrospinal fluid, or a sterile body fluid. Positive eye and skin cultures, and patients aged less than 72 hours and more than 90 days of age were excluded. The medical records of the included patients were studied after obtaining the Investigational Review Board (IRB) approval.

\section{Results}

During our study period, 223 patients were identified to have LOS, with peak incidence in 2014 (Figure 1). We identified a total of 213 organisms. Ninety-eight organisms were identified as gram positive organisms (46\%) compared to 104 (48.8\%) and 11 (5.2\%) as gram negative organisms and fungi respectively (Figure 2). Among gram positive organisms, CONS were most prevalent at $52 \%(n=51)$, followed by Staphylococcus aureus at rate of $14.2 \%(n=14)$. As for gram negative organisms, Klebsiella pneumoniae was most prevalent at $43.2 \%(n=45)$, followed by E.coli at $21.2 \%(n=22)$, and third by Pseudomonas at $10.6 \%$ $(n=11)$ (Figure 3). Assessment of our regimen's adequacy revealed $100 \%$ sensitivity to Vancomycin among gram positive organisms and $95.5 \%$ sensitivity to Gentamicin among gram negative organisms.

\section{Discussion}

LOS in NICU is considered a nosocomial infection, which results in prolonged hospitalization and increased risk of mortality in unfortunate circumstances. Worldwide incidences of gram positive and gram-negative organisms in LOS are variable among regions, hospitals and patients' demographics. Not only that, but the incidence seems to change from time to time in the same region and hospital. Boghossian NS et al. conducted a retrospective study in United States looking at 15178 singleton and 5294 multiple gestation infants. The authors reported an estimated incidence of $25 \%$ among singleton births and $22.6 \%$ among multiple births of very low birth weight infants [4]. In our study the rate was $8.9 \%$ over the complete study period. Although we did not specifically categorize our patients to singleton and multiple gestations, our rate of LOS seems to be lesser all together than what has been reported in United States.

The most common organism among the gram positive ones was CONS at a rate of $52 \%(n=51)$ and among the gram negative ones was Klebsiella Pneumoniae at a rate of $43.2 \% \quad(n=45)$ (Figure 3). Generally, throughout the study period it has been noted that gram negatives were the leading causative organisms, nevertheless their contribution to LOS etiology was trending down in our unit (Figures 1 and 3).

Units around the world use different antibiotics regimens for empirical LOS therapy. Among the most common combinations used was the combination of Flucloxacillin and Gentamicin, Ampicillin and Gentamicin or Vancomycin with either Cefotaxime or Gentamicin.

The rate of gentamicin resistance is increasing across the regions. In some Asian centers gentamicin resistance was found to be as high as $37 \%$ while it was around $14.7 \%$ in our neighbouring country Kuwait [6-8]. Over our study period, the total percentage of gentamicin resistance was $4.5 \%$. Such high sensitivity to this empirical regimen supports our choice for the time being.

The rise of Extended Spectrum Beta-lactamase (ESBL) organisms is a serious threat due to the wide use of broadspectrum antibiotics. Yilmaz et al. compared the rate of ESBL organisms between 2004 and 2008 in Turkey. In their review, ESBL organisms increased from $14 \%$ to $33 \%$ for E.coli; and from $30 \%$ to $44 \%$ for Klebsiella spp. over 2004 and 2008 respectively [7-9]. Our ESBL rate among gram negative organisms during our study period was $19.2 \%(n=20)$ which constituted only $9.4 \%$ of the total organisms contributing to LOS with a notable increasing trend over the years (Figures 4 and 5). Therefore an empirical coverage for ESBL organisms doesn't seem to be needed in our unit at this time.

Invasive fungal infections in neonates require vigilant assessment due to unfavourable long-term outcome and prolonged length of therapy. Dong Y. and Speer C. looked at candida spp. in LOS different parts of the world [6,10-12]. Two of our neighbouring countries (Turkey and Kuwait) were included in their review. They revealed approximate rates of $28 \%$ and $12 \%$ in Turkey and Kuwait respectively, while in our study the rate of fungi contributing to LOS was $5.2 \%$ (Figure 2). Based on those comparative rates, we believe that empirical antifungal therapy is not indicated in our unit at this point [13-16].

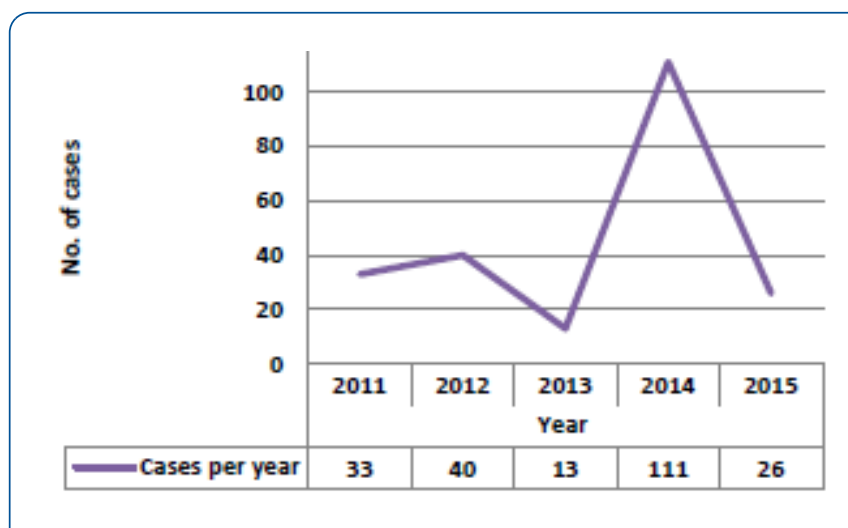

Figure 1: Cases of late onset sepsis per year.

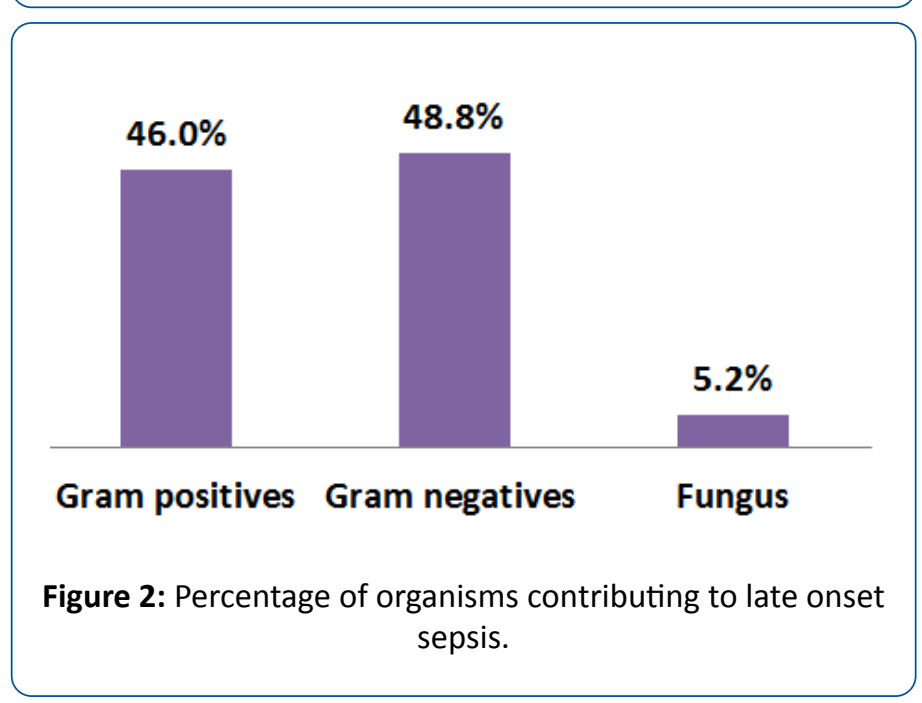




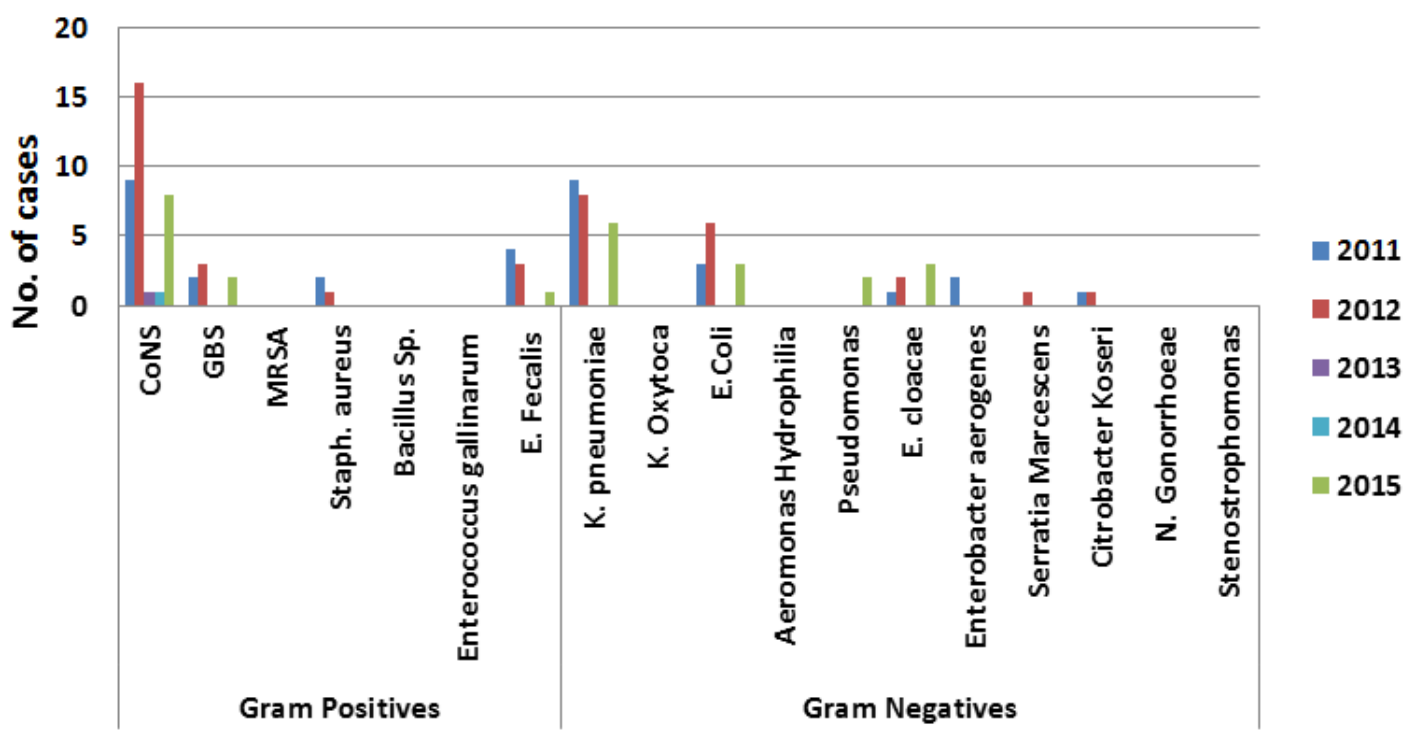

Figure 3: Microrganisms contributing to late onset sepsis.

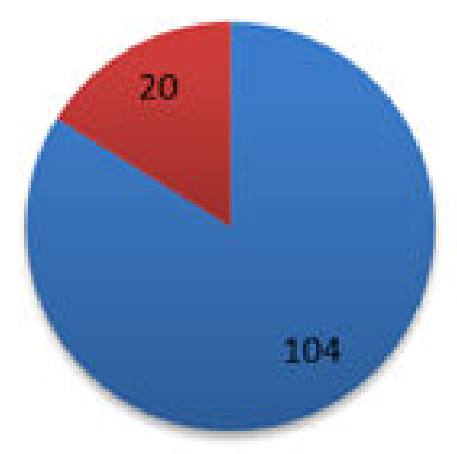

Eram Negatives ESBL

Figure 4: ESBLs among gram negative organisms.

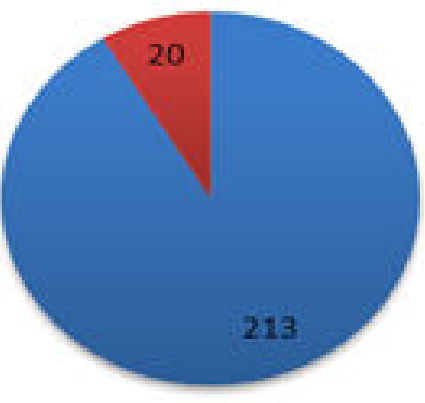

= Total Organisms = ESBL

Figure 5: ESBLs among all organisms.

\section{Conclusion}

In our study, we found out that our rate of LOS was less than what has been reported in some countries like United States. However, the overall spectrum of causative organisms was almost similar to worldwide reports. In our unit, we use Vancomycin and Gentamicin as the empirical choice in treating LOS. Our study revealed that the current regimen provided sufficient coverage for the vast majority of LOS causative organisms over our study period. Our current ESBL organisms and fungi infections do not prompt a shift in current management or addition of empirical antifungal respectively. Still we believe that the local biograms must be revisited from time to time to optimize empirical antibiotic choices and ensure a better quality of care for our patients.

\section{References}

1. IM S (2011) Neonatal sepsis. Biochem Med (Zagreb) 21: 276-281.

2. Wynn J, Wong H (2010) Pathophysiology and Treatment of Septic Shock in Neonates. Clin Perinatol 37: 439-479.

3. Camacho-Gonzalez A, Spearman P, Stoll B (2013) Neonatal Infectious Diseases: Evaluation of Neonatal Sepsis. Pediatr Clin North Am 60: 367-389.

4. Boghossian (2013) Late-Onset Sepsis in Very Low Birth Weight Infants from Singleton and Multiple Gestation Births. J Pediatr 162: $1120-1124$.

5. Long S, Pickering L, Prober C (2012) Principles and Practice of Pediatric Infectious Diseases. $4^{\text {th }}$ edn. Edinburgh: Elsevier Churchill Livingstone.

6. Dong Y, Speer C (2014) Late-onset neonatal sepsis: recent developments. Arc Dis Child Fetal Neonate edn 100: F257-F263.

7. Yilmaz N, Agus N, Helvaci M, Kose S, Ozer E, et al. (2010) Change in Pathogens Causing Late-onset Sepsis in Neonatal Intensive Care Unit in Izmir, Turkey. Iran J Pediatr 20: 451-458. 
8. Van den Hoogen A, Gerards L, Verboon-Maciolek M, Fleer A, Krediet $T$ (2010) Long-Term Trends in the Epidemiology of Neonatal Sepsis and Antibiotic Susceptibility of Causative Agents. Neonatology 9: 22-28.

9. Lahra M, Beeby P, Jeffery H (2009) Intrauterine Inflammation, Neonatal Sepsis, and Chronic Lung Disease: A 13-Year Hospital Cohort Study. Pediatrics 123: 1314-1319.

10. Al-Taiar A, Hammoud M, Cuiqing L, Lee J, Lui K, et al. Neonatal infections in China, Malaysia, Hong Kong and Thailand. Arc Dis Child Fetal Neonate edn 98: F249-F255.

11. Tröger B, Göpel W, Faust K, Müller T, Jorch G, et al. (2014) Risk for Late-onset Blood-culture Proven Sepsis in Very-low-birth Weight Infants Born Small for Gestational Age. Pediatr Infect Dis J 33: 238-243.

12. Morioka I, Morikawa S, Miwa A, Minami H, Yoshii K, et al. (2012) Culture-Proven Neonatal Sepsis in Japanese Neonatal Care Units in 2006-2008. Neonatology 102: 75-80.
13. Leal Y, Álvarez-Nemegyei J, Velázquez J, Rosado-Quiab U, DiegoRodríguez N, et al. (2012) Risk factors and prognosis for neonatal sepsis in southeastern Mexico: analysis of a four-year historic cohort follow-up. BMC Pregnancy Childbirth 12: 1.

14. Cogen A, Nizet V, Gallo R (2008) Skin microbiota: a source of disease or defence? Br J Dermatol 158: 442-455.

15. Vergnano $\mathrm{S}$, Menson $\mathrm{E}$, Kennea $\mathrm{N}$, Embleton $\mathrm{N}$, Russell $\mathrm{A}$, et al. (2010) Neonatal infections in England: the Neonatal surveillance network. Arc Dis Child Fetal Neonate edn 96: F9-F14.

16. Shim G, Kim S, Kim H, Kim E, Lee $H$, et al. (2011) Trends in Epidemiology of Neonatal Sepsis in a Tertiary Center in Korea: A 26-Year Longitudinal Analysis, 1980-2005. J Korean Med Sci 26: 284. 